\title{
Direct observation of dynamic shear jamming in dense suspensions
}

Ivo R. Peters ${ }^{\text {a) }}$, Sayantan Majumdar, Heinrich M. Jaeger

James Franck Institute, The University of Chicago, Chicago, IL, 60637, USA

a) i.r.peters@ soton.ac.uk; Current address: Engineering and the Environment, University of Southampton, Highfield, Southampton SO17 1BJ, UK

Liquid-like at rest, dense suspensions of hard particles can undergo striking transformations in behaviour when agitated or sheared ${ }^{1}$. These phenomena include solidification during rapid impact ${ }^{2,3}$, as well as strong shear thickening characterized by discontinuous, orders of magnitude increases in suspension viscosity ${ }^{4-8}$. Much of this highly non-Newtonian behaviour has recently been interpreted within the framework of a jamming transition. However, while jamming indeed induces solid-like rigidity $^{9-11}$, even a strongly shear-thickened state still flows and thus cannot be fully jammed ${ }^{12,13}$. Furthermore, while suspensions are incompressible, the onset of rigidity in the standard jamming scenario requires an increase in particle density ${ }^{9,10,14}$. Finally, while shear thickening occurs in the steady state, impact-induced solidification is transient $^{15-17}$. As a result, it has remained unsettled how these dense suspension phenomena are related and how they are connected to jamming. Here we resolve this by systematically exploring both the steady-state and transient regimes with the same experimental system. We demonstrate that a fully jammed, solid-like state can be reached without compression and instead purely by shear, as recently proposed for dry granular systems ${ }^{18,19}$. In contrast to dry granular materials, however, this state is created by transient shear-jamming fronts, which we track for the first time directly. We also show that shear stress, rather than shear rate, is the key control parameter. From these findings we map out a state diagram with particle density and shear stress as variables. Discontinuous shear thickening is newly identified with a marginally jammed regime just below the onset of full, solid-like jamming ${ }^{20}$. This state diagram provides a new, unifying framework, compatible with prior experimental and simulation results on dense suspensions, that connects steady-state and transient behaviour in terms of a dynamic shear-jamming process.

Jamming transitions transform fluid-like particle systems into amorphous solids with finite yield stress when the particle packing fraction $\phi$ increases beyond a critical value, $\phi_{J}$. In the standard scenario ${ }^{10}$, the jammed state is reached via isotropic compression, and for frictionless particle interactions the jammed system will weaken and eventually unjam when shear stress is applied. In suspensions, on the other hand, shear can play the opposite role by inducing viscosity increases and even solidification. Here the idea has been that shear reorganizes particles into anisotropic configurations that form large clusters and potentially a load bearing network. With frictionless, purely hydrodynamic interactions between suspended particles, such 'hydroclusters'121,22 can, however, only give rise to mild increases in viscosity (continuous shear thickening $)^{6,13,23}$. Frictional contacts are required to produce the large jumps in viscosity associated with strong, discontinuous shear thickening (DST) $)^{6,7,13,24,25}$. How shear can convert isotropic unjammed particle configurations below $\phi_{J}$ into anisotropic jammed configurations was shown explicitly for dry granular systems by $\mathrm{Bi}$ et $a{ }^{18}$ if the particle interactions are frictional and by Kumar and Luding for the frictionless case ${ }^{19}$. This introduced to the original jamming phase diagram a new regime of shear-jamming, which subsequently has been adopted as a candidate mechanism for $\mathrm{DST}^{6,8,13}$. However, key conceptual as well as experimental questions have remained open. In particular, DST occurs under steady-state shearing conditions and thus cannot involve an actual solid-like, jammed response. Importantly, the onset stress for DST is known to be essentially independent of packing fraction ${ }^{5}$, while the predicted onset stress for shearjamming decreases and reaches zero as $\phi_{J}$ is approached ${ }^{18}$. Furthermore, direct observations of solid-like shear-jamming under controlled conditions have so far been in quasi-static systems of dry grains ${ }^{18,19}$. In other situations where suspensions appear to jam fully, e.g. during impact-induced solidification, the conditions typically are more complex and in principle can involve shear as well as compression ${ }^{2,3,17}$.

To sort this out requires experiments on dense suspensions where solidification fronts can be generated solely by shear and where also DST can be observed. We achieve this here by using a Couette-type geometry. Our results show, for the first time, how solidification fronts produce a solid-like shearjammed state. This state is qualitatively different from DST in that it exhibits a yield stress, which we demonstrate explicitly by showing how it prevents weights from sinking in. Our findings suggest that DST does not correspond to a jammed but instead to a fragile state, which exhibits intermittent flow that can be thought of as a precursor to shear-jamming and exhibits behaviour of marginal material that is neither freely flowing nor fully jammed ${ }^{20}$.

The experiments were performed using a large gap between the inner and outer wall of the cylindrical cell filled with the suspension, which allowed us to directly observe both the transient and steady-state velocity profiles by imaging from above with a high-speed camera (Fig. 1). The suspensions consisted of density-matched solutions of water, glycerol, and $\mathrm{CsCl}$, mixed with cornstarch, at packing fractions $\phi$ from 0.43 by volume up to values close to the isotropic $\phi_{J}$ for this material. A key feature of our experiment was that by rotating the inner cylinder in this Couette-type geometry we were applying only shear, eliminating any compression which could result in a significant increase in packing fraction. 

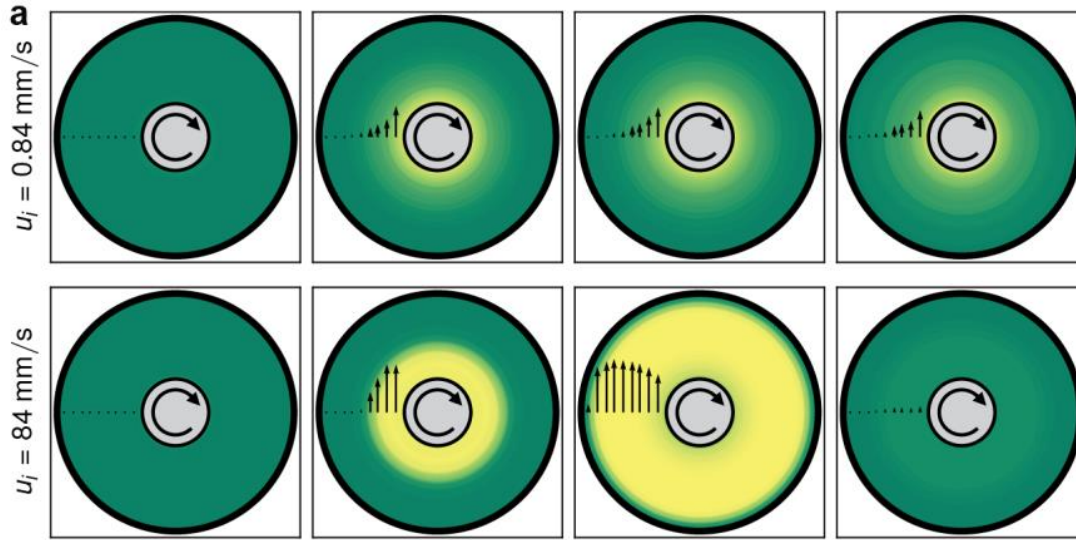

$\theta=0.0$

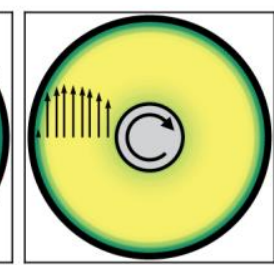

$\theta=0.2$

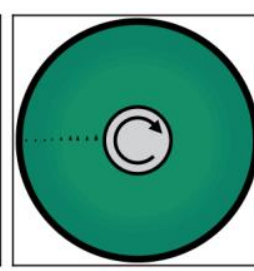

$\theta=0.3$
1.00

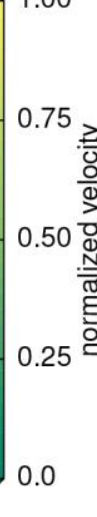

b

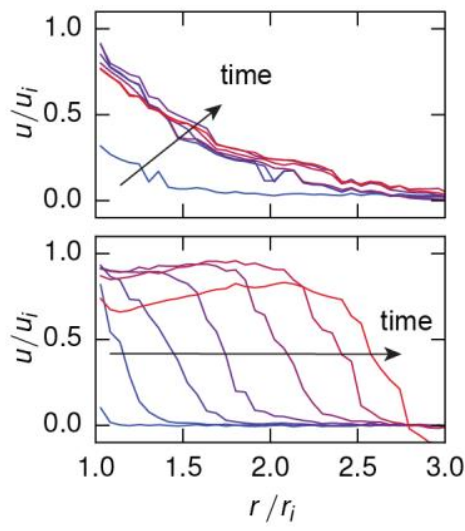

Figure 1 | Transition from viscous response to rapid front propagation. a, Evolution of the radial velocity profile, obtained from particle image velocimetry, for two different driving speeds: Top row $0.84 \mathrm{~mm} / \mathrm{s}$, and bottom row $84 \mathrm{~mm} / \mathrm{s}$ (100 times faster). Time is increasing from left to right. Data for both driving speeds are shown for the same amount of shear strain, given by the rotation angle $\theta$. All velocities are normalized by the driving speed $u_{i}$ at the outer edge of the inner cylinder. Where slow driving results in a velocity profile that gradually changes until it reaches a fluid-like steady state, fast driving shows the formation of a traveling jamming front and eventually a solid-like shearjammed state (bottom row, far right). b, Velocity versus radial distance corresponding to the same two driving speeds as in a. The radial distance $r$ is normalized by the radius $r_{i}$ of the inner cylinder. Different curves represent different instances in time, with time increasing from blue to red. The steady-state diffusive profile (top) and the traveling front (bottom) are clearly apparent.

Starting with the suspension at rest, sudden rotation of the inner cylinder initiates jamming fronts that propagate radially outward, converting fluid-like into solid-like material, similar to what is observed for impact normal to a free suspension surface $^{2,17}$. To illustrate the effect of rate dependence on this transient response we show in Fig. 1 the evolution of the velocity field for two different rotation speeds. We find strikingly different behaviour between slow and fast driving of the inner cylinder. For a slow driving speed (top), we observe a velocity profile that behaves in a diffusive manner, reminiscent of that of a viscous liquid, where the spreading slows down as time progresses and monotonically approaches an equilibrium profile (which is similar to those measured by Fall et $a l .^{8}$ ). At high driving speeds a sharp front develops (bottom), which rapidly travels radially outward. Behind the front the velocity of the suspension is fairly uniform, while ahead of the front the suspension is still at rest. After the front reaches the outer wall (bottom right in Fig. 1a), the system fully jams while the inner cylinder continues rotating, creating a plug of solid-like material between inner and outer cylinder.

When fronts develop, their speed is proportional to the driving speed, as shown in Fig. 2a. Rescaling the x-axis by the driving speed collapses the data (Fig. 2b), indicating that the ratio between driving speed and front propagation speed is independent of the driving speed. The absence of any time scale indicates that the front propagation is resulting only from a critical shear strain, which locally shear-jams the system, not unlike neighbouring gears that engage. Once this critical strain has been reached between any two adjacent radial layers of the suspension, the now shear-jammed portion will be able to strain the still unjammed suspension ahead of it. This process will continue for as long as the system is actively driven and no boundary is reached. The closer the packing density is to the jamming point, the smaller will be the critical strain required to reach a shear-jammed state ${ }^{18}$. This implies that the propagation speed will increase with packing fraction, as shown explicitly in Fig. 2 b.

We can take the connection between front propagation in suspensions and shear jamming of dry granular systems one important step further by accounting for a key feature of the shear-jamming phase diagram introduced by $\mathrm{Bi}$ et $a{ }^{18}$, namely that both critical stress and strain approach zero as $\phi$ approaches $\phi_{J}$. Such vanishing critical strain would, according to our reasoning above, result in a diverging front speed, as an infinitesimal perturbation could immediately propagate through the whole system. The functional form of how the critical strain approaches zero, and consequently how the speed diverges as $\phi_{J}$ is approached is not known. Our data, however, can be approximated by the same functional dependence $u_{f} / u_{i}=\left(\phi_{0} /\left(\phi_{J}-\phi_{0}\right)\right)^{\alpha}$ derived for speeds of compression-induced fronts in dry granular materials close to the jamming point ${ }^{26}$. Here $u_{f}$ is the front speed and $u_{i}$ the driving, or impact, speed. In the original expression for compression fronts, the exponent $\alpha=1$. Treating $\alpha$ and $\phi_{J}$ as fitting parameters, we find $\alpha=1.0 \pm 0.1$ also for shear-jamming fronts, and we obtain an estimated $\phi_{J} \approx 0.56$.

Figure $2 \mathrm{~d}$ shows that there is a critical driving speed beyond which the torque response jumps by several orders of magnitude, much like in a discontinuous shear thickening (DST) transition. Right at the transition (red curve), the torque wanders off, which shows that the transition itself cannot be resolved in a rate-controlled manner. We therefore turn to stress-controlled measurements to address the central remaining question, namely how this shear-jammed state relates to the well-established steady-state properties of dense suspensions ${ }^{27}$. In previous work, the transient and steady state behaviour typically were investigated in different systems and by very different methods, since fast transient responses involving front velocities of a few $\mathrm{m} / \mathrm{s}$ (Fig. 2a) are difficult to track with standard rheological experiments. In our wide-gap Couette cell, however, we can readily investigate both regimes.

Figures 3a-b show the steady-state suspension viscosity $\eta^{*}$ versus applied shear stress $\tau$ for two of the packing fractions investigated. Note that $\eta^{*}$ is the apparent viscosity, defined as ratio of shear stress to shear rate (see Methods). From the slope of the curves in plots like these we identify the following behaviours: Newtonian, shear thinning, weak shear thickening, and Discontinuous Shear Thickening (DST). Specifically, the DST regime is identified in the most strict 

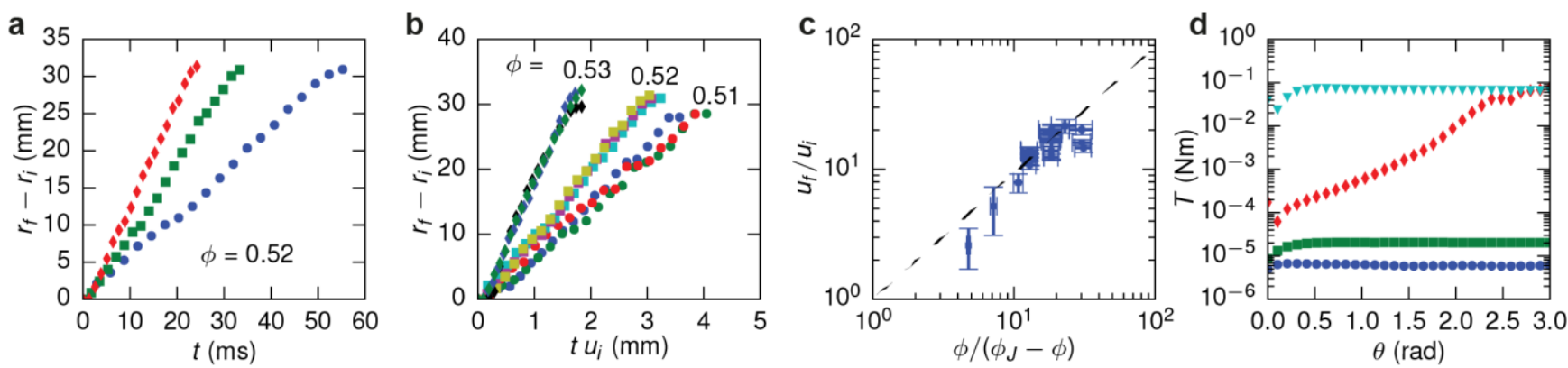

Figure 2 | Influence of driving speed and packing fraction. a, Radial position of the shear front (defined as the position where $u=u / 2$ as a function of time for different driving speeds (blue circles: $u_{i}=59 \mathrm{~mm} / \mathrm{s}$, green squares: $u_{i}=92 \mathrm{~mm} / \mathrm{s}$, red diamonds: $u_{i=1}=126 \mathrm{~mm} / \mathrm{s}$ ), for packing fraction $\phi=0.52$. Here $r_{i}$ is the radius of the inner cylinder. $\mathbf{b}$, Front positions normalized by driving speed (different driving speeds indicated by different colours) for different packing fractions (circles: $\phi=0.51$, squares: $\phi=0.52$, diamonds: $\phi=0.53$. The collapse of data shows that the jamming front propagates due to a critical shear strain. c, Front speed $u_{i}=d r_{f} / d t$ over driving speed ratio vs packing fraction. The black dashed line has a slope $\alpha=1.0$, showing that the speed ratio is approximately proportional to $\left.\phi / \phi_{s} \phi\right)$. Error bars indicate the standard deviation of repeated experiments. d, Measured torque $T$ for different, fixed driving speeds. The blue circles $84 \mu \mathrm{m} / \mathrm{s})$ and green squares $(0.84 \mathrm{~mm} / \mathrm{s})$ correspond to the behaviour shown in the upper half of Fig. 1. Cyan triangles $(84 \mathrm{~mm} / \mathrm{s})$ correspond to the front formation shown in the bottom row of Fig. 1. The 3-4 orders of magnitude larger torque signals that the front has reached the container wall and a fully jammed, solid-like state has been established (the torque asymptotes because of slip at the wall of the inner, driven cylinder). Red diamonds ( $8.4 \mathrm{~mm} / \mathrm{s})$ show the situation right at the transition to front formation. Here the suspension goes through the DST regime into a fully shear-jammed state as the applied torque increases while the driving speed is kept constant.

sense, i.e., by a linear or stronger increase of $\eta^{*}$ with $\tau$. We determine the onset of DST by a linear fit to the DST regime on log-log plots like Fig. 3a, and find the stress value at which it intersects a fit to the Newtonian regime, where the viscosity is constant, independent of applied stress.

At low $\phi$ around 0.43 , the typical sequence of behaviours with increasing $\tau$ within our experimental range moves from shear-thinning via Newtonian to DST (Fig. 3a). At large $\phi$ around 0.51 (Fig. 3b), the onset of DST is sharper and DST crosses over into a fully shear-jammed (SJ) state that no longer flows and behaves like a solid. In this SJ regime the inner, rotating cylinder continually slips and the value of $\eta^{*}$ is no longer meaningful.

We demonstrate the solid behaviour in the SJ regime explicitly by dropping small steel spheres (diameter $5.0 \mathrm{~mm}$ ) onto the continuously sheared suspension (Fig. 3c) and tracking their vertical position after they touch the surface (Fig. 3d). As we apply more shear stress to our system, the trajectory of the spheres changes from slowly sinking (unjammed or DST) to rebounding and remaining on the suspension surface for as long as shear-stress is applied (shear-jammed). We define the SJ onset stress by the suspension behaving elastically, i.e., by the applied shear stress at which the spheres' velocity reverses direction after impact (see Methods).

By performing these experiments across a range of packing fractions below $\phi_{J}$ we construct the state diagram shown in Fig. 3e. Its main feature is the delineation between a strongly shear-thickening (DST) regime and a solid-like, shear-jammed (SJ) regime. As we move towards lower packing fractions, DST is partially replaced by weaker shear thickening. This agrees with prior work that shows DST eventually disappearing at even lower $\phi^{28}$.

We point out an important difference with previous studies that suggested to identify DST directly with the shear jamming transition ${ }^{6,8,13}$. These studies found that the minimum shear rate for observing DST approaches zero as $\phi$ approaches
$\phi_{J}$. However, the shear jamming phase diagram, just as the original jamming phase diagram, is controlled by packing density and shear stress, not rate, as shown in Fig. $2 \mathrm{c}$ of $\mathrm{Bi}$ et $a l .{ }^{18}$. In fact, other experiments have shown that the onset stress $\tau_{\min }$ for DST is independent of packing fraction, and, if anything, even increases on the approach to the isotropic jamming point (due to an emerging yield stress at high packing fractions) ${ }^{12}$. The behaviour of the onset stress for DST therefore would seem to be in direct contradiction to the shear-jamming phase diagram. As seen in Fig. 3e, this issue is easily resolved by recognizing the DST regime as a precursor to shear jamming transition, with a lower boundary that is essential independent of $\phi$ (see Methods for the slightly different definition of $\tau_{\min }$ here and in prior work).

In different experimental geometries, such as parallel plate setups, an upper limit to DST was observed ${ }^{28}$, due to the maximum confinement available from surface tension and given by $\tau_{\max } \sim 0.1 \sigma / a$. Here $\sigma$ is the interfacial tension at the free suspension boundary and $a$ the particle diameter. We find that this limit does not apply with our setup since we see no significant difference when $\sigma \rightarrow 0$ (see Methods). It implies that both the flowing DST state and the fully jammed SJ state are highly anisotropic, and any force network must lie predominantly within the horizontal shear plane. Since in the absence of friction such networks would immediately break up since particles would slide out of plane, we interpret this anisotropy as a signature of the highly frictional particle contacts that enable shear-jamming.

This new identification of DST is, to the best of our knowledge, consistent with all previous studies and it reframes DST within the context of shear-jamming. In particular, the pronounced downward curvature of the upper boundary of DST as $\phi$ approaches the isotropic jamming point at $\phi_{J}=0.56$ follows exactly the qualitative behaviour pointed out by $\mathrm{Bi}$ et al. ${ }^{18}$ and thus gives the first clear indication of a state diagram that unifies DST with shear jamming. 

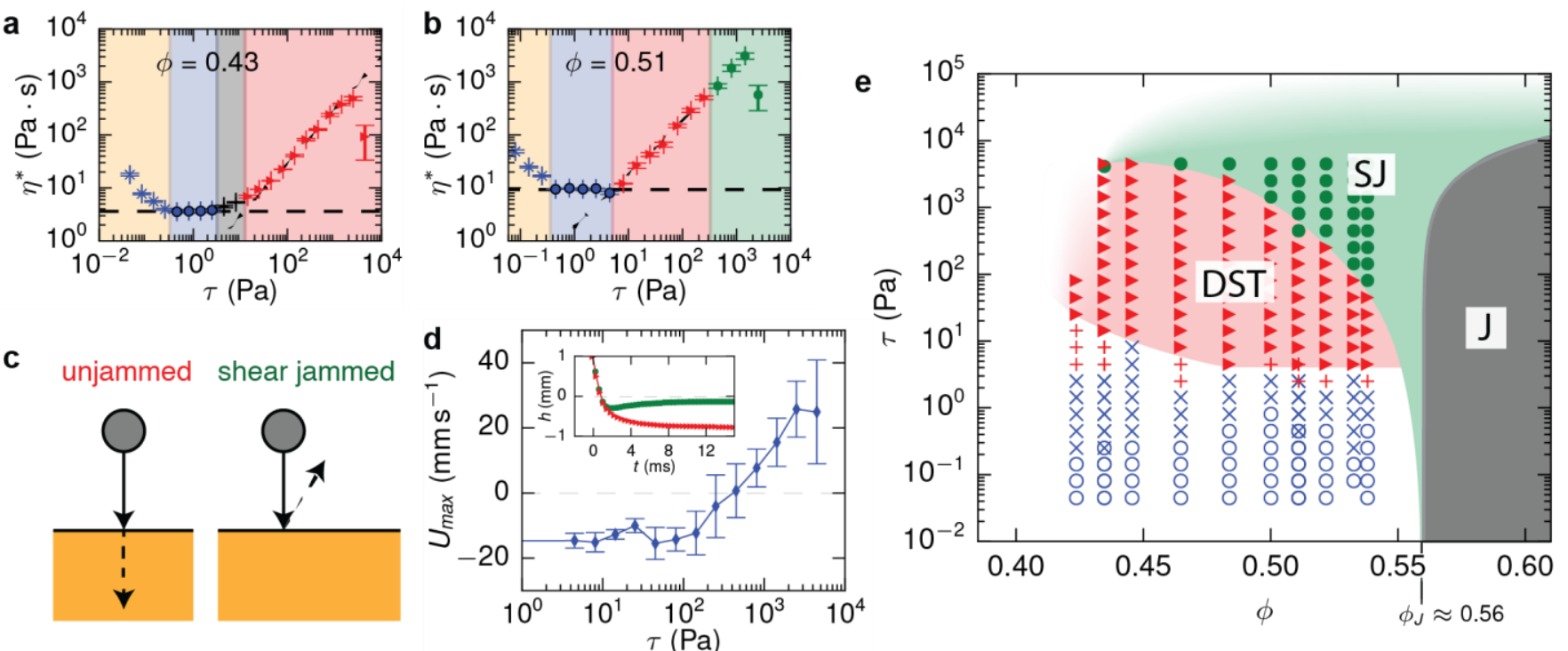

C
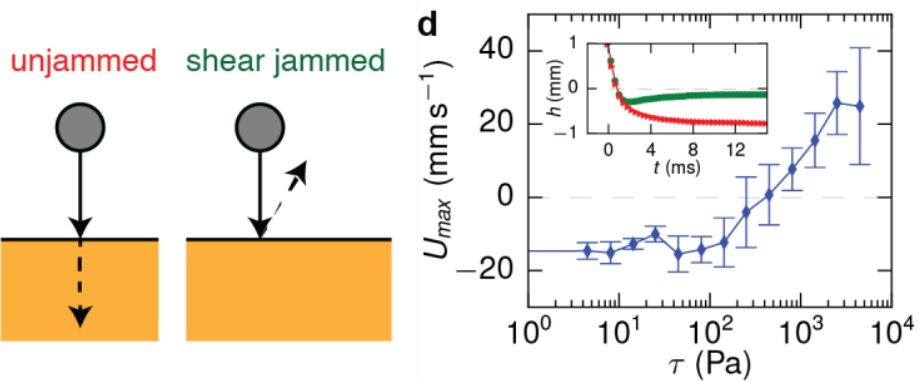

Figure 3 | Transitions between the different states of the suspension. $\mathbf{a}, \mathbf{b}$, Viscosity-stress curves for two different packing fractions, with different states indicated by different background colours: shear thinning (beige), Newtonian (blue), weak shear thickening (grey), discontinuous shear thickening (red), and shear jammed (green). At high packing fractions, the weaker shear thickening state becomes negligible. Error bars are standard deviations of the temporal fluctuations in viscosity. Note that both data sets show one data point where slip occurs on the inner cylinder (on the far right), which results in a much lower apparent viscosity. Clearly, these data points are not suitable to determine an effective viscosity. c, Cartoon of the impact experiment. d, Transition curve for the cross-over from DST to shear jamming, showing the maximum observed upward velocity $U_{\max }$ of the sphere after impact as a function of applied shear stress. Error bars give the standard deviation obtained from repeated experiments. Inset: two experimental trajectories of spheres impacting on the suspension under two different shear stresses, showing a slowly sinking sphere in the DST regime (red curve) and rebound in the shear jammed regime (green curve). Here, $h$ is the vertical distance between the bottom of the sphere and a fixed reference point in the high-speed movies. e, State diagram showing the different states of the suspension: shear thinning (blue circles), Newtonian (blue crosses), weaker shear thickening (red crosses), discontinuous shear thickening (red triangles) and shear jammed (green dots). The shaded areas correspond to the original shear jamming state diagram ${ }^{18}$, with isotropic jammed (J) and shear jammed (SJ). The regime of fragile states is here identified with the DST regime. The regime of shear thinning or Newtonian behaviour at stresses below the onset of DST does not exist in dry granular systems, and in suspensions corresponds to conditions where stresses are too low to allow frictional particle-particle contacts ${ }^{6,7}$

Our findings suggest the following picture. At low shear stress, particles are not in contact and lubrication forces result in a Newtonian behaviour (or shear thinning in case of a yield stress). Beyond a critical stress, lubrication breaks down, and frictional forces generate a fabric of force chains, resulting in discontinuous shear thickening ${ }^{6,13}$. However, rather than fully jammed, this is a fragile state that intermittently flows and gets stuck. This required critical stress is independent of packing fraction because it only depends on the microscopic breakdown of lubrication ${ }^{6,13,24}$. With increasing stress, the force network becomes denser until a fully shear jammed state is reached. This last transition depends strongly on the packing fraction, as the critical shear stress will vanish on the approach to the isotropic jamming point ${ }^{18}$. We point out that these shear jammed states emerge without any change in packing fraction. This is in contrast with the initial picture developed for solidification under impact ${ }^{2}$, which was based on a change in $\phi$ and needs to be reconsidered within a shearjamming framework. Finally, our observation of discontinuous shear-thickening states without a transition to shear-jamming suggests that, at low packing fractions, fragile states are possible, but will fail at higher stresses before a shear jammed state is reached. These transitions may possibly be explored in dry granular systems using photoelastic particles.

1. Barnes, H. A. Shear-Thickening ('Dilatancy') in Suspensions of Nonaggregating Solid Particles Dispersed in Newtonian Liquids. J. Rheol. 33, 329-366 (1989).

2. Waitukaitis, S. R. \& Jaeger, H. M. Impact-activated solidification of dense suspensions via dynamic jamming fronts. Nature 487, 205209 (2012).

3. Petel, O. E. et al. The effect of particle strength on the ballistic resistance of shear thickening fluids. Appl. Phys. Lett. 102, 064103 (2013).
4. Fall, A., Huang, N., Bertrand, F., Ovarlez, G. \& Bonn, D. Shear Thickening of Cornstarch Suspensions as a Reentrant Jamming Transition. Phys. Rev. Lett. 100, 018301 (2008).

5. Brown, E. \& Jaeger, H. M. Dynamic Jamming Point for Shear Thickening Suspensions. Phys. Rev. Lett. 103, 086001 (2009).

6. Seto, R., Mari, R., Morris, J. F. \& Denn, M. M. Discontinuous Shear Thickening of Frictional Hard-Sphere Suspensions. Phys. Rev. Lett. 111, 218301 (2013).

7. Wyart, M. \& Cates, M. E. Discontinuous shear thickening without inertia in dense non-brownian suspensions. Phys. Rev. Lett. 112, 098302 (2014).

8. Fall, A. et al. Macroscopic Discontinuous Shear Thickening versus Local Shear Jamming in Cornstarch. Phys. Rev. Lett. 114, 098301 (2015).

9. Cates, M., Wittmer, J., Bouchaud, J.-P. \& Claudin, P. Jamming, Force Chains, and Fragile Matter. Phys. Rev. Lett. 81, 1841-1844 (1998).

10. Liu, A. J. \& Nagel, S. R. Jamming is not just cool any more. Nature 396, 21-22 (1998)

11. Trappe, V., Prasad, V., Cipelletti, L., Segre, P. N. \& Weitz, D. A. Jamming phase diagram for attractive particles. Nature 411, 772775 (2001)

12. Brown, E. et al. Generality of shear thickening in dense suspensions. Nat. Mat. 9, 220-224 (2010).

13. Mari, R., Seto, R., Morris, J. F. \& Denn, M. M. Shear thickening, frictionless and frictional rheologies in non-Brownian suspensions. J. Rheol. 58, 1693-1724 (2014).

14. Keys, A. S., Abate, A. R., Glotzer, S. C. \& Durian, D. J. Measurement of growing dynamical length scales and prediction of the jamming transition in a granular material. Nat. Phys. 3, 260264 (2007).

15. Liu, B., Shelley, M. \& Zhang, J. Focused Force Transmission through an Aqueous Suspension of Granules. Phys. Rev. Lett. 105, 188301 (2010).

16. von Kann, S., Snoeijer, J., Lohse, D. \& van der Meer, D. Nonmonotonic settling of a sphere in a cornstarch suspension. Phys. Rev. E 84, 060401 (2011).

17. Peters, I. R. \& Jaeger, H. M. Quasi-2D dynamic jamming in cornstarch suspensions: visualization and force measurements. Soft Matter 10, 6564-6570 (2014).

18. Bi, D., Zhang, J., Chakraborty, B. \& Behringer, R. P. Jamming by 
shear. Nature 480, 355-358 (2011).

19. Kumar, N. \& Luding, S. Memory of jamming - multiscale flow in soft and granular matter. (2015). arXiv:1407.6167.

20. Vitelli, V. \& van Hecke, M. Marginal matters. Nature 480, 325-326 (2011).

21. Wagner, N. J. \& Brady, J. F. Shear thickening in colloidal dispersions. Phys. Today 62, 27-32 (2009).

22. Cheng, X., McCoy, J. H., Israelachvili, J. N. \& Cohen, I. Imaging the microscopic structure of shear thinning and thickening colloida suspensions. Science. 333, 1276-1279 (2011).

23. Lin, N. Y. C. et al. Hydrodynamic and Contact Contributions to Continuous Shear Thickening in Colloidal Suspensions. Phys. Rev. Lett. 115, 228304 (2015).

24. Fernandez, N. et al. Microscopic Mechanism for Shear Thickening of Non-Brownian Suspensions. Phys. Rev. Lett. 111, 108301 (2013).

25. Guy, B. M., Hermes, M. \& Poon, W. C. K. Towards a Unified Description of the Rheology of Hard-Particle Suspensions. Phys. Rev. Lett. 115, 088304 (2015).

26. Waitukaitis, S. R., Roth, L. K., Vitelli, V. \& Jaeger, H. M. Dynamic jamming fronts. Europhys. Lett. 102, 44001 (2013).

27. Brown, E. \& Jaeger, H. M. Shear thickening in concentrated suspensions: phenomenology, mechanisms and relations to jamming. Rep. Prog. Phys. 77, 046602 (2014).

28. Brown, E. \& Jaeger, H. M. The role of dilation and confining stresses in shear thickening of dense suspensions. J. Rheol. 56, 875-923 (2012).

Acknowledgements We thank Eric Brown, Endao Han, Nicole James, Shomeek Mukhopadhyay and Qin Xu for stimulating discussions. This work was supported by the US Army Research Office through grant W911NF-12-1-0182 and the Chicago MRSEC, which is funded by NSF through grant DMR-1420709. S.M. acknowledges support through a Kadanoff-Rice fellowship.

Author Contributions I.R.P., S.M. and H.M.J. conceived the project, I.R.P. and S.M. performed the experiments and the analysis, I.R.P. and H.M.J. wrote the manuscript.

Author Information The authors declare no competing financial interests.

\section{METHODS}

Experimental setup. We used an Anton Paar MCR301 rheometer with a Couette-type cell consisting of two concentric cylinders where the outer cylinder (cup) was a glass container with inner diameter of $98 \mathrm{~mm}$ and height of 42 $\mathrm{mm}$. The cup was filled just below the rim with the suspension. The inner cylinder (bob) of diameter of $26.7 \mathrm{~mm}$ was controlled by the rheometer and was partially submerged in the suspension. We verified that the measurements were not influenced by the bottom of the cup by performing experiments at different insertion depths of the bob. In addition we performed tests where we effectively removed the solid bottom boundary by using a layer of fluorinert (FC-3283) on which the suspension floated. At high stresses, we observed slip between the surface of the bob and the suspension. We also performed experiments with a roughened cylinder surface (using sandpaper), but this still resulted in slip at the bob surface, and had no significant influence on our measurements.

Transient Behaviour. For these experiments the rheometer was set to rotate at constant speed. The time to accelerate from rest was always much smaller than the time during which we observed the transient response of the system. The experiments were recorded using a high-speed camera (Phantom V9), imaging at rates up to 4500 frames/sec, depending on the driving speed. Tracer particles (ground black pepper) sprinkled on the suspension surface of the cornstarch suspension allowed us to perform Particle Image Velocimetry (PIV) and obtain time-resolved velocity fields (e.g., Fig. 1 and Supplementary Information Video 2). In addition, we measured the torque applied by the rheometer.

Steady-State Behaviour. These experiments were performed by setting a constant torque and measuring the rotation speed as a function of time. We repeated this for a range of torques to obtain viscosity-stress curves. We calculated the stress as $\tau=T /\left(2 \pi R_{i}^{2} h\right)$, with $T$ the torque, $R_{i}$ the radius of the bob and $h$ the submerged height of the bob. The local shear rate $\dot{\gamma}$ is a priori unknown (an example of the flow profile at low stress is given in the top row of Fig. 1b). We define an average shear rate via the relation $\dot{\gamma}=\omega\left(R_{o}^{2}+R_{i}^{2}\right) /\left(R_{o}^{2}-R_{i}^{2}\right)$ in order to calculate the apparent viscosity $\eta^{*} / \dot{\gamma}$, with $\omega$ the angular rotation rate and $R_{o}$ the radius of the outer cylinder. Such apparent viscosity corresponds to the true viscosity only for a linear, Newtonian suspension. In order to calculate the local apparent viscosity everywhere in the system, stress gradients would need to be taken into account in order to obtain the local stress. However, the current method suffices to identify transitions between different states of the suspension with changes in applied shear stress. From the resulting curves of viscosity versus shear stress we define the onset of DST as the intersection of fits to Newtonian and DST regimes as described in the main text. Prior work defined the onset of general (weak or strong) shear thickening via $\tau_{\min }$, the minimum in viscosity-stress curves before the start of thickening. Taking this as the criterion in Fig. 3c, we see that even at lower packing fractions the onset stress for shear thickening remains independent of $\phi$. To test for the upper stress limit $\tau_{\max } \sim 0.1 \sigma / a$, identified as the stress scale above which DST is no longer observable in parallel-plate geometries because confinement breaks down, we performed experiments where we flooded the top free surface of our suspension with a liquid that is miscible with the suspending liquid, a procedure which effectively sets the interfacial tension to zero. In this flooded system, fully shear-jammed, solid-like behaviour was still observed, and stress levels exceeding $\tau_{\max }$ could easily be achieved. This demonstrates that suspensions driven into a frictional jammed state by (horizontal) shear do not develop significant out-of-plane (vertical) stresses that would need to be balanced by confinement via boundaries or interfacial tension.

Determining the Onset of Shear-Jamming. For the impact experiments we released steel spheres with diameter $5 \mathrm{~mm}$ and mass $0.51 \mathrm{~g}$ from a height of $95 \mathrm{~mm}$, see Supplementary Information Video 1. An electromagnetic release mechanism was used to ensure reproducible impact conditions. The impacts were recorded with the high-speed camera described above, and analysed using a particle tracking algorithm. Each experiment was performed up to 10 times to obtain a distribution of responses for every combination of packing fraction and shear stress. We note that under our experimental conditions the impacting sphere merely probes the state of the suspension and does not cause it to undergo a transition into a jammed configuration.

In order to determine if a system is jammed, we ideally need to probe if the system has a yield stress. We here use an elastic response (rebound) as a proxy to having a yield stress, because a rebound can be determined more precisely and unambiguously in experiments. We do, however, point out that also for longer times the yield stress behaviour is apparent. An example of this can be observed in Supplementary Information Video 1 and Extended Data Figure 2 where a sphere sits on the surface of a suspension for several seconds, until the supplied stress is turned off. 
Suspension Packing Fraction. The suspensions consisted of cornstarch dispersed in a density-matched solution of water, glycerol, and $\mathrm{CsCl}$. The cornstarch was stored at controlled temperature $\left(22.8 \pm 0.3^{\circ} \mathrm{C}\right)$ and relative humidity $(51 \pm 2 \%)$. For each solution, we carefully measured the density and liquid viscosity. In order to calculate the packing fraction of the suspensions, we took into account the porosity of the cornstarch particles. In considering jamming, the relevant quantity is the amount of free interstitial volume available for particle rearrangement. If some of the interstitial liquid penetrates into porous particles the available free volume shrinks. Therefore, a meaningful parameter is the effective packing fraction, which considers the interior as inaccessible to other particles. Assuming that each particle has a volume fraction $\lambda=0.3$ (see, e.g., Refs. ${ }^{29,30}$ ) of pore space, we can write $\phi=(1+\lambda) \phi_{v}$, where $\phi_{v}$ is the material packing fraction without accounting for pore space, and $\phi$ the packing fraction we quote in this paper. The value of $\lambda$ is an estimate; but it only adjusts the absolute values of $\phi$ and has no qualitative influence on our results. In order to calculate $\phi_{v}$, we take into account the moisture content $(\beta)$ which the cornstarch has absorbed from the environment. Assuming that this moisture content is pure water, we calculate the packing fraction as:

$$
\phi_{v}=\frac{(1-\beta) m_{c S} / \rho_{c s}}{(1-\beta) m_{c s} / \rho_{c S}+m_{l} / \rho_{l}+\beta m_{c s} / \rho_{w}}
$$

with $\rho_{c s}=1.62 \cdot 10^{3} \mathrm{~kg} / \mathrm{m}^{3}$ the density of the dry cornstarch, $\rho_{l}$ and $m_{l}$ the density and mass of the water/glycerol/CsCl solution, $m_{c s}$ the mass of the cornstarch including the moisture content, and $\rho_{w}$ the density of pure water. The moisture content is estimated to be $\beta=0.1$, but small variations will result in small variations in the packing fraction, which influence the experiments significantly only when very close to the isotropic jamming point $\phi_{J}$. We found that for experiments which were performed within a relatively short time frame ( days), we can assume a constant value for $\beta$. The value for $\beta$ can be determined by performing a set of experiments at different packing fractions and determining the jamming packing fraction, which should be the same for all experiments. Sets of experiments that were performed when the moisture content had changed, could be matched to the existing data sets by adjusting $\beta$ accordingly.

Influence of solvent viscosity. The ratio between propagation speed of the jamming fronts $u_{f}$ and the driving speed $u_{i}$ depend on the packing fraction of the suspension. Because the jamming front is a result of frictional interaction between the particles, this ratio $u_{f} / u_{i}$ is expected to be independent of the solvent viscosity if the shear stress is high enough ${ }^{2,31}$. To test this we performed a number of experiments where we kept the packing fraction constant, and only changed the solvent viscosity. The results are shown in Extended Data Fig. 1a, where we plot the ratio $u_{f} / u_{i}$ as a function of the solvent viscosity. We similarly tested the influence of solvent viscosity on the onset of bouncing motion for the impact experiments. For this, we used two different viscosities, as can be seen in Extended Data Fig. 1b. Note from the error bars that in this case the data is less noisy for the more viscous fluid (green data points), which gives a more accurate determination of the onset for bouncing.

29. Sair, L. \& Fetzer, W. R. Water Sorption by Starches. Ind. Eng. Chem. 36, 205-208 (1944).

30. Hellman, N. N. \& Melvin, E. H. Surface Area of Starch and its Role in Water Sorption. J. Am. Chem. Soc. 72, 5186-5188 (1950).

31. Waitukaitis, S. R. Impact-Activated Solidification of Cornstarch and Water Suspensions. (Springer International Publishing, 2014). doi:10.1007/978-3-319-09183-9 

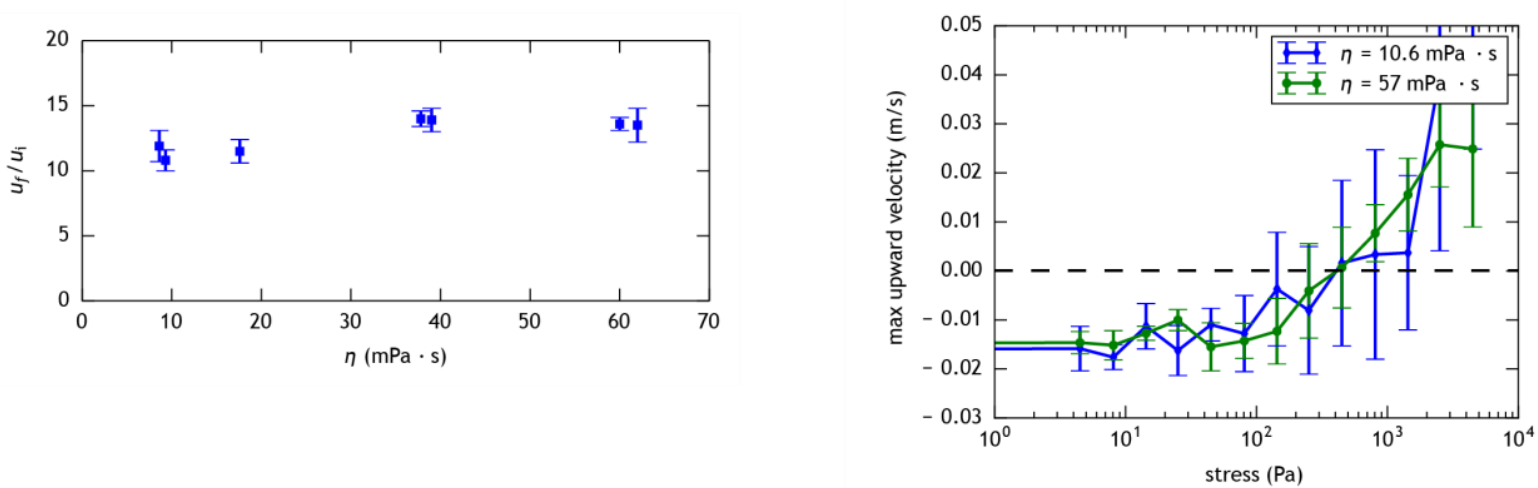

Extended Data Figure 1 | Influence of viscosity on front speed and shear jamming transition. a, Ratio between front speed and driving speed as a function of the solvent viscosity. Error bars are standard deviations of repeated experiments at different driving speeds $u_{i}$. $\mathbf{b}$, Bouncing transition curves for two different solvent viscosities. The horizontal axis gives the applied shear stress, the vertical axis the maximum upward velocity we observed from the trajectory of the impacting sphere. Error bars are standard deviations of repeated experiments. 


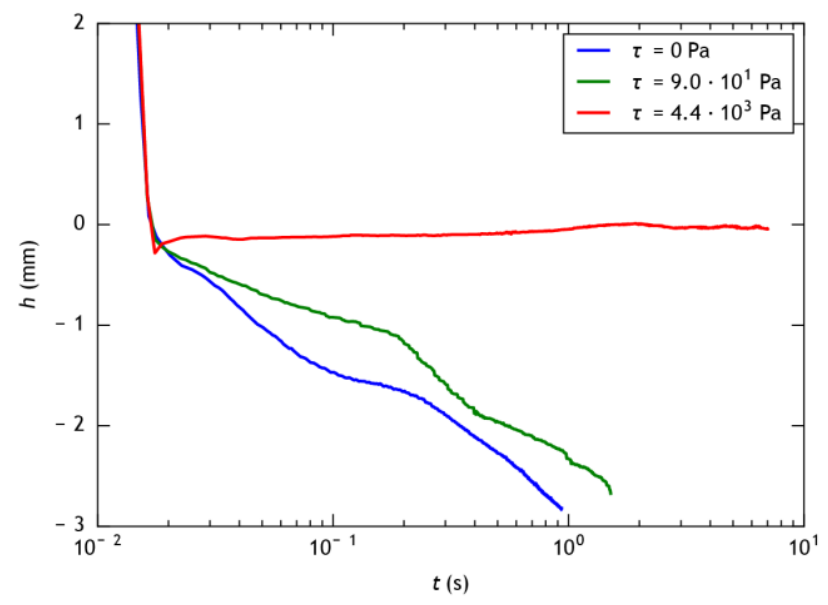

Extended Data Figure 2 | Long-time behaviour of shear jammed and unjammed suspension. Experimental trajectories showing the long-time behaviour of spheres impacting on the suspension under three different shear stresses. Zero shear stress (blue curve) and a stress of $90 \mathrm{~Pa}$ (DST, green curve) both show a slowly sinking sphere. The shear jammed state (4400 Pa, red curve) shows a rebound followed by yield stress behaviour, evident by the sphere not sinking in. 